\title{
Higher-Order and Modal Logic as a Framework for Explanation-Based Generalization
}

\author{
Scott Dietzen and Frank Pfenning \\ School of Computer Science \\ Carnegie Mellon University \\ Pittsburgh, Pennsylvania 15213-3890 \\ To appear: Sixth International Workshop on Machine Learning \\ June 1989
}

\begin{abstract}
Logic programming provides a uniform framework in which all aspects of explanation-based generalization and learning may be defined and carried out, but first-order Horn logic is not well suited to application domains such as theorem proving or program synthesis where functions and predicates are the objects of computation. We explore the use of a higher-order representation language and extend EBG to a higher-order logic programming language. Variables may now range over functions and predicates, which leads to an expansion of the space of possible generalizations. We address this problem by extending the logic with the modal $\square$ operator (indicating necessary truth) which leads to the language $\lambda^{\square}$ Prolog. We develop a meta-interpreter realizing EBG for $\lambda^{\square}$ Prolog and give some examples in an expanded version of this extended abstract which is available as a technical report [2].
\end{abstract}

\section{Introduction}

To date, work on the explanation-based approach to learning relies primarily on explanation-based generalization (EBG) as its central mechanism $[8,1]$. Recently, the logic programming paradigm has been used as a foundation for supporting EBG [7, 11, 5]. One argument put forward in favor of the logic programming framework is that it admits a uniform representation for all aspects of EBG: domain theory, training instance, goal, goal concept, operationality definition, etc. This helps in explicating the underlying principles in a uniform way and clarifies semantic issues.

Generalization and learning performance are intimately tied to the underlying language for representation, or representation domain. If knowledge is encoded in an inappropriate representation domain, then it is less likely that the desired generalizations can be expressed in a natural and concise manner, and also less likely that they can be found.

In this paper we explore two ways of enriching the representation domain of Horn logic, while staying within the logic programming framework: integrated support for higher-order objects including variables ranging over such objects (functions and predicates) and support for modal concepts. Both of these have a significant impact on EBG. We concentrate on how a rich representation language supports EBG, and largely ignore questions about how these generalizations may be assimilated and applied automatically. For a discussion of these issues, see Prieditis [11], and Donat \& Wallen [4]. We also do not deal with the important concept of operationality; see Hirsh [5] for a treatment in the context of logic programming.

We begin by motivating the step to a higher-order language as the representation domain. We then address the intractability of the space of possible higher-order generalizations by differentiating domain theory and training instance in a 'logical' way. As $\lambda$ Prolog is not initially suited to supporting this distinction, we extend the language with the modal operator $\square$ forming $\lambda^{\square}$ Prolog. We then develop an implementation of EBG within $\lambda^{\square}$ Prolog through a generalizing meta-interpreter written in $\lambda$ Prolog. 
Acknowledgments. Our meta-interpreter depends on eLP, the implementation of $\lambda$ Prolog developed by Conal Elliott in the framework of the Ergo Project at Carnegie Mellon University. We thank Conal Elliott and Haym Hirsh for their thoughtful comments on our presentation. This research was supported in part by the Office of Naval Research under contract N00014-84-K-0415 and in part by the Defense Advanced Research Projects Agency (DOD), ARPA Order No. 5404, monitored by the Office of Naval Research under the same contract.

\section{Higher-order EBG and Representation Domains}

A domain is higher-order if it contains higher-order values such as functions or predicates. A language or application over such a domain is itself said to be higher-order. For example, a higher-order programming language allows functions to be bound to variables, passed as parameters, and returned from function calls. Similarly, a higher-order logic provides for quantification over functions and predicates. Higher-order EBG is explanation-based generalization in which the candidates for variable replacement include higher-order objects.

A representation domain is a language for expressing application domain values. When higher-order domains are expressed in first-order languages, reasoning and programming over these ad hoc encodings is difficult: We often need "new variables", need to check conditions such as "where ... does not occur in ...", or must implement substitution in a way that "renames bound variables if necessary." In this paper we explore the use of higher-order representation domains that provide for natural expression and manipulation of higherorder objects. Consider the following higher-order rule for reducing an integration task by extracting a constant factor $a$ :

$$
\forall a \forall f \forall g . \quad \int f=g \Rightarrow \int \lambda x . a * f(x)=\lambda x . a * g(x)
$$

( $\lambda$ is used to explicitly express function abstraction.) Within a higher-order representation domain, that is, one that supports name binding (via $\lambda$ ), the restriction that $x$ not be free in the expression $a$ is captured without complicating side conditions.

Many other domains naturally involve binding constructs and are thus best expressed in a higher-order representation domain: logics (when viewed as domains), programming languages, and natural language [10, 9]. Similar representational needs arise when one wants to reason "at the meta-level", that is, about control strategies for logic programming, theorem proving, or the EBG algorithm itself: One would like facts (propositions) or properties (predicates) to be objects themselves. Prolog and other first-order languages allow this to some extent, but in a way that is only operationally, but not logically motivated. This complicates reasoning about these concepts and, in practice, often prohibits applications such as EBG.

Nadathur \& Miller describe $\lambda$ Prolog, a logic programming language supporting higher-order functions and predicates [9]. It is based on higher-order hereditary Harrop formulas which generalize Horn clauses while still preserving the basic properties of the logic programming paradigm. $\lambda$ Prolog serves simultaneously as our representation domain and as the implementation language for EBG.

\section{EBG and Modal Logic}

When describing domain theory and training instance, we distinguish the former with the $\square$ operator. is borrowed from modal logic (see, for example, Hughes \& Cresswell [6]). It precedes necessarily true sentences, or equivalently, those true in all worlds; non-prefixed sentences are only contingently true, or true in the current world. Previous EBG implementations have differentiated between domain theory and training instance either by explicit separation [8] or by distinguishing rules (implications) from facts (atomic clauses) $[7,5,11]$. A modal logic representation subsumes the latter and can also be given a clear semantics 
which is independent of a particular generalization algorithm or search procedure. We illustrate the use of $\square$ with a first-order example from [1]:

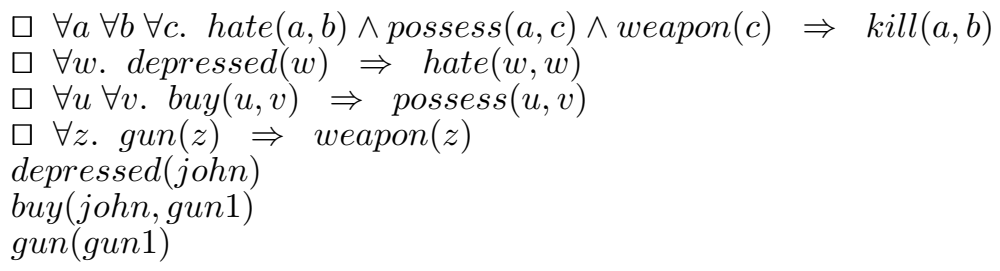

The generalization resulting from the query $k i l l(j o h n$, john) may be expressed as

$$
\forall a \forall b \text {. depressed }(a) \wedge \operatorname{buy}(a, b) \wedge \operatorname{gun}(b) \Rightarrow \operatorname{kill}(a, a)
$$

Clauses of the training example (i.e., those without a $\square$ prefix) can safely be removed without invalidating the generalization. Revising or replacing the training instance can be explained semantically as switching worlds. Since $\square$ is not restricted to outermost occurrences, one has the potential to intermix domain and training knowledge.

The higher-order and modal aspects of our representation domain work well together: Through the use of $\square$ to distinguish domain theory and training example, we elegantly restrict the space of generalizations for higher-order EBG. This generalization space is much larger than that for first-order EBG, since functions and predicates are also candidates for variable replacement. Our higher-order EBG algorithm is sufficient to determine goal concepts for published examples (such as the above) and allows direct application to higher-order domains such as theorem proving and program derivation.

As we have made $\square$ part of our logic, it must become part of the underlying logic programming language. The expanded language, $\lambda^{\square}$ Prolog, is implemented with an interpreter written in $\lambda$ Prolog. The concise and elegant extension of this interpreter to perform EBG along the lines of [7, pages 387-388] is presented in full in $[2]$.

\section{References}

[1] Gerald DeJong and Raymond Mooney. Explanantion-based generalization: an alternate view. Machine Learning, $1(2): 145-176,1986$.

[2] Scott Dietzen and Frank Pfenning. Higher-Order and Modal Logic as a Framework for Explanation-Based Generalization. Technical Report CMU-CS-89-160, Carnegie Mellon University, Pittsburgh, June 1989.

[3] Scott Dietzen and William L. Scherlis. Analogy in program development. In J. C. Boudreaux, B. W. Hamill, and R. Jernigan, editors, The Role of Language in Problem Solving 2, pages 95-117, North-Holland, 1987. Also available as Ergo Technical Report 86-013, Carnegie Mellon University, Pittsburgh.

[4] Michael R. Donat and Lincoln A. Wallen. Learning and applying generalised solutions using higher order resolution. In Ewing Lusk and Ross Overbeek, editors, 9th International Conference on Automated Deduction, Argonne, Illinois, pages 41-60, Springer-Verlag LNCS 310, Berlin, May 1988.

[5] Haym Hirsh. Explanation-based generalization in a logic-programming environment. In Proccedings of IJCAI, pages 221-227, 1987.

[6] G. E. Hughes and M. J. Cresswell. An Introduction to Modal Logic. Methuen and Co., Ltd., London, 1968.

[7] Smadar T. Kedar-Cabelli and L. Thorne McCarty. Explanantion-based generalization as resolution theorem proving. In Proceedings of the Fourth International Workshop on Machine Learning, pages 383-389, 1987.

[8] Tom M. Mitchell, Richard M. Keller, and Smadar T. Kedar-Cabelli. Explanantion-based generalization: a unifying view. Machine Learning, 1(1):47-80, 1986.

[9] Gopalan Nadathur and Dale Miller. An overview of $\lambda$ Prolog. In Robert A. Kowalski and Kenneth A. Bowen, editors, Logic Programming: Proceedings of the Fifth International Conference and Symposium, Volume 1, pages 810-827, MIT Press, Cambridge, Massachusetts, August 1988.

[10] Frank Pfenning and Conal Elliott. Higher-order abstract syntax. In Proceedings of the SIGPLAN '88 Symposium on Language Design and Implementation, Atlanta, Georgia, pages 199-208, ACM Press, June 1988. Available as Ergo Report 88-036.

[11] Armand E. Prieditis and Jack Mostow. Prolearn: toward a Prolog interpreter that learns. In Proceedings of $A A A I$, Spring 1987. 